\title{
Affluence vs. Destitution
}

\author{
By N.N.
}

- Desideratum

\section{Evernote keywords: issues tagged for first-priority treatment in this entry}

- academic unemployment

- basic needs azmah / survival

- bread $(Q T h)$

- children

- credit cards

- déclassement social

- deprivation

- dire living conditions

- drinking water

- freedom of access to culture

- gas $(Q T h)$, gasoline

- gated communities

- gold

- golf

- hard currency manipulation

- health Minister, health problems/risk

- heart diseases, heart surgery

- high prices

- hospitals (QTh)

- housing projects, social housing program

- IMF

- imports, import of cars, imported, import-export

- industry

- infitāh (Open Door politics)

- inflation, increase of inflation, prices of daily needs raising, increase of lemon prices (during Ramaḍān), of electricity prices

- informal economy

- investment

- irrigation

- job losses, job market

- Kuwait

- Labour Day

- lack of development

- lack of savings
- layoffs

- the less well-off

- loans

- luxury

- malls

- al-Mahalla al-Kubrà

- Manshiyat Nașr

- market, marketing

- meat crisis

- middle class

- money

- money cartoon

- monetary crisis

- nouveaux riches, parvenus

- Panama leak, Panama papers

- panic

- pashas

- pensions

- Philip Rizq

- poison

- popular unrest

- population growth

- poverty

- privileges

- production, productivity

- proprietors

- prostitution

- public office

- Pyramids

- quality of education

- real estate

- revenge

- rich people, Rich vs. Poor

- salaries

- Sayyidnā al-Ḥusayn

- shouting

- slums

- small shops 
- soap operas (musalsalāt)

- social classes

- social effects

- social conditions/poverty, inequality
- social indignation, social unrest

- social (in)justice (QTh), inequality

- visitors

- water supply

Entries pointing here

ARRAYS - Baby Milk $\downarrow$ Clash $\downarrow$ Disappearances $\downarrow$ Disasters $\downarrow$ Dual Identities $/$ Masking $\downarrow$ Father Figures Garbage Gated Communities / Compounds Hashish $\downarrow$ Language $\star$ Mobile Phones The Policeman Criminal Pop Music $\downarrow$ The Voice from Above

CODES - Beautiful $v s$. Ugly $\downarrow$ Security $v s$. Fear $\diamond$ True $v s$. False

CODES COLLAPSED - True $=$ False $($ Life in Limbo $)$ 